\title{
Signal Amplification and Control in Optical Cavities with Off-Axis Feedback
}

\author{
Roberta Zambrini* \\ Institute for Cross-Disciplinary Physics and Complex Systems, IFISC (CSIC-UIB), Palma de Mallorca, 07122, Spain \\ Francesco Papoff \\ Department of Physics, University of Strathclyde, 107 Rottenrow, Glasgow G4 ONG, United Kingdom
}

(Received 16 April 2007; published 9 August 2007)

\begin{abstract}
We consider a large class of optical cavities and gain media with an off-axis external feedback which introduces a two-point nonlocality. This nonlocality moves the lasing threshold and opens large windows of control parameters where weak light spots can be strongly amplified while the background radiation remains very low. Furthermore, transverse phase and group velocities of a signal can be independently tuned and this enables us to steer it nonmechanically, to control its spatial chirping, and to split it into two counterpropagating ones.
\end{abstract}

DOI: 10.1103/PhysRevLett.99.063907

PACS numbers: 42.65.Sf, 42.55. $-\mathrm{f}$, 89.75.Kd

In this Letter we study the effects introduced by a twopoint nonlocality [1] on a broad class of nonlinear equations with both diffusion and diffraction. Systems modeled by these types of equations can be experimentally realized in optics by cavities with an off-axis external feedback, which is the spatial analog of a feedback with temporal delay [2]. Off-axis feedback has been the subject of theoretical and experimental study in liquid crystal light valves [3], Kerr-like media [4,5], and generic nonlinear diffusive systems [6]. Here, instead, we consider a broad class of optical cavities using a general formalism that can be applied to gases, solid states, and semiconductor media with fast decay of the polarization, including media with a negative refractive index and devices with soft apertures. The simultaneous presence of diffusive and diffractive terms appears in universal Ginzburg-Landau equations describing the behavior of any spatially extended system near the onset of oscillations, such as, for instance, reaction-diffusion systems and lasers [7]. These equations also describe properties of systems with time-delayed feedback and no spatial degrees of freedom when the delay is an order of magnitude larger than the other time scales [8], with the slow time formally taking the role of the spatial variable. We show that the inclusion of a two-point nonlocality generalizes these equations introducing new regimes, and is a powerful way to amplify, characterize, and control perturbations, either external or intrinsic to the system. In particular, nonlocality changes the nature of the first instability, which without nonlocality leads to a spatially extended, lasing state. With nonlocality, on the contrary, there are large windows of control parameters where small localized signals can be strongly amplified while the background radiation in other region of the system remains very low. Furthermore, the signal moves across the cavity with transverse phase and group velocities that are easily managed to have the same or opposite signs. It is indeed possible, without altering the mechanical alignment of the setup, to control signal motion, tuning continuously the group velocity so that a localized perturbation is steered either towards or against the offset direction and can even be split into two counterpropagating components. The tunability of the phase velocities allows us to control the spatial chirping of light signals independently from the direction of steering. These unusual properties open new possibilities for light control and can underpin applications in optical communications, imaging, and micromanipulation.

In the following, we analyze how the first threshold depends on nonlocality, diffusion, and diffraction; determine the nature of the instability; find a second threshold; and derive the equations for the phase and group velocity of localized perturbations. We consider optical systems described by nondimensional equations of the type

$$
\begin{aligned}
& \partial_{t} E=g_{1}\left(|E|^{2}, N ; \mu\right) E+e^{i \delta} \partial_{x x}^{2} E+r e^{i \phi} E_{\Delta x}, \\
& \partial_{t} N=g_{2}\left(|E|^{2}, N, \partial_{x x}^{2} N ; \mu\right)
\end{aligned}
$$

where $E$ is the complex slowly varying amplitude of the electric field, $N$ is the population inversion, and $\mu$ is a control parameter. We consider one transverse dimension $x$ as nonlocality changes only the spatial dependence of the dispersion along the direction of the shift. Time and space are scaled with field decay and with the square root of the modulus of the Laplacian coefficient. Our analysis encompasses devices with diffraction and diffusion due to Fourier filtering by intracavity soft apertures [9] or to elimination of the fast variables [10], as well as media with positive or negative refractive index [11]. $\delta$ gives the relative strength of diffusion and diffraction, with $\delta \in(0, \pi / 2)$ for positive refractive indexes and $\delta \in(-\pi / 2,0)$ for negative indexes, corresponding to left-handed materials. The term $r e^{i \phi} E_{\Delta x}$ represents nonlocal coupling of the field $E$ in a point $x$ with the field $E_{\Delta x}$ in a point $x+\Delta x$ and is the consequence of an off-axis, single-passage feedback loop. This is characterized by an amplitude $0<r<1$ and a phase shift $\phi$ accumulated by the fast component of the electric field in 

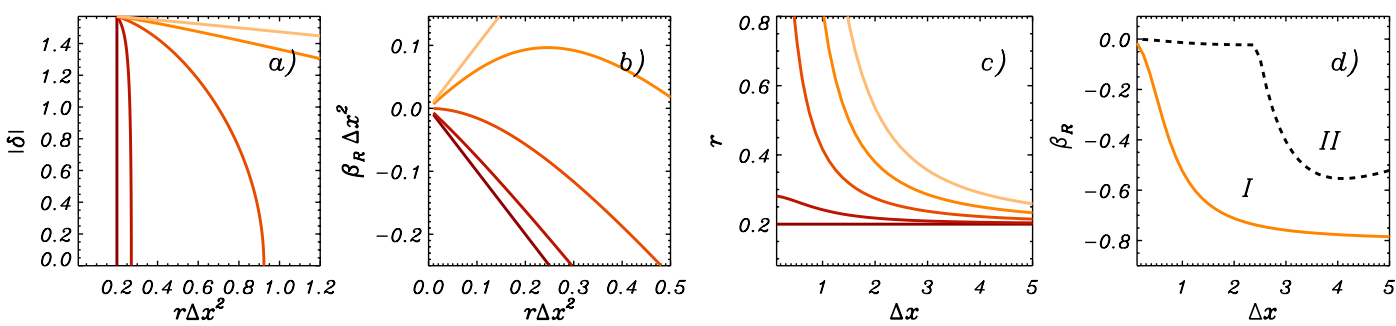

FIG. 1 (color online). (a) Instability thresholds for $\beta_{R} \Delta x^{2}=-0.2$ and for $\phi=n \pi / 4$ with $n=0,1,2,3,4$ (from dark to light colors). The lowest threshold is found for $\phi=0$ and the instability takes place on the right of the lines. (b) Thresholds for $\delta=0.45 \pi$ and different feedback $\phi$ as in (a). (c) Thresholds for $\beta_{R}=-0.2, \delta=0.45 \pi$ and different feedback $\phi$ as in (a). (d) First (continuous line) and second (dashed line) thresholds for $\delta=0.45 \pi, r=0.8$, and $\phi=\pi / 2$.

the external loop. We assume that the temporal delay of the feedback is negligible compared to the time scales of $E$ and $N$. The generic complex functions $g_{1,2}$ allow us to describe all class $B$ lasers, including semiconductor. The following analysis immediately applies also to (i) the simpler case of systems in which the variable $N$ can be eliminated (class $A$ ) and (ii) a more general class of equations in which the feedback term is nonlinear [12].

We consider perturbations $\delta E \propto \exp (\omega t+i k x)$ of the nonlasing solution $E_{0}=0$ and $N_{0}$ such that $g_{2}\left(0, N_{0}\right)=0$. These perturbations have complex dispersion relation

$$
\omega=\beta-e^{i \delta} k^{2}+r e^{i(\phi+k \Delta x)}
$$

with $\beta=g_{1}\left(0, N_{0} ; \mu\right)$ also complex. In the following, real and imaginary parts of complex quantities have subindices $R$ and $I$, respectively. In the limit of vanishing shift $\Delta x=$ 0 , the laser threshold, given by $\beta_{R}^{\text {th }}=-r \cos (\phi)$, decreases when the feedback interferes constructively with the intracavity field and increases when the interference is destructive. Because the fast relaxation of the polarization implies that the gain bandwidth is very large, all traveling waves have the same gain or loss if there is no diffusion. The effect of diffusion is to filter the high Fourier components so that the most unstable mode is the homogeneous one $(k=0)$ independently from the relative strength of diffusion and diffraction $(\delta)$. When $\Delta x \neq 0$, on the other hand, the most unstable mode can have $k \neq 0$. The nonlocality gives rise to a modulation instability and allows for the existence of several bands of unstable wave vectors $\left(\omega_{R}>0\right)[3]$.

The off-axis feedback, besides modulation instability to several bands of wave vectors, provides a wide tunability of the properties of the device and enables to control the first threshold. Inspection of Eq. (2) shows that the instability threshold can be expressed as a function of four relevant parameters, namely $\phi, \delta, r \Delta x^{2}$, and $\beta_{R} \Delta x^{2}$ [see Figs. 1(a) and 1(b)]; therefore increasing the shift size $\Delta x$ produces on the device the same effect of larger gain $\beta_{R}$ and feedback $r$. As a specific effect of the nonlocality, we find that the relative strength of diffusion and diffraction, $\delta$, also becomes an effective parameter to control the threshold position. Indeed, the lowest gain and feedback thresholds (independently on the feedback phase $\phi$ ) are generally found in the purely diffractive limit $(\delta \sim \pi / 2)$. The effect of diffusion on the feedback lasing threshold can be appreciated in Fig. 1(a): for any not vanishing feedback phase $\phi$, the threshold value for the scaled feedback strength $r \Delta x^{2}$ increases with the diffusion, being independent on the sign of the refractive index (sign of $\delta$ ). Both $\beta_{R}$ and $r$ can be increased to cross the laser threshold as shown in Fig. 1(b), and-similarly to the case of perfect alignment - if the feedback is out of phase then stronger gain is required. For fixed values either of the gain or of the feedback the nonlocality strongly decreases the threshold values for the gain as well as for the feedback field, as seen in Figs. 1(c) and 1(d). This can be understood considering that the most unstable mode has $k \neq 0$ so that the effect of the nonlocal coupling is equivalent to a reduction of the feedback dephasing. Consistent with this interpretation, in the case of feedback perfectly in phase with the intracavity field $(\phi=0)$, the threshold is independent on the lateral shift $\Delta x$ because the most unstable mode is the homogeneous one $(k=0)$.

Another effect of the nonlocality concerns the possibility to tune transverse phase and group velocities independently from one another. This property, which is absent in purely diffusive systems [6,13], enables nonmechanical steering and spatial chirping of light beams as the high spatial frequencies can accumulate in the left or right side of the beam. We remark that, as for conventional lasers without off-axis feedback [14], phase traveling waves are exact solutions of the model. Phase and group velocities follow from Eq. (2):

$$
\begin{gathered}
v_{p}=-\frac{\omega_{I}(k)}{k}=k \sin \delta-\frac{\beta_{I}+r \sin (k \Delta x+\phi)}{k}, \\
v_{g}=-\partial_{k} \omega_{I}=2 k \sin \delta-r \Delta x \cos (k \Delta x+\phi) .
\end{gathered}
$$

They can be tuned independently because the parameter $\beta_{I}$ enters only in the expression of the phase velocity. Evaluation of the velocities for the critical wave vectors $k_{c}$ allows us to identify the manifolds in the control parameter space that separate regions in which the group and the phase velocity have the same sign from the region in which these velocities have opposite sign. In particular, the group velocity is null for $r \Delta x^{2}=-2 \delta \pm(4 n+1) \pi \mp$ $2 \phi$. As shown in Fig. 2 equal or opposite signs of the 

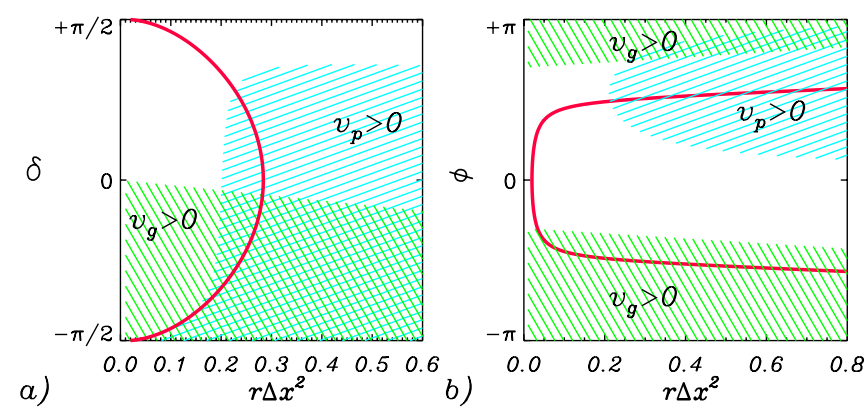

FIG. 2 (color online). Sign of velocities for $\beta \Delta x^{2}=-0.02-$ $i 0.2$, and for $\phi=\pi / 2$ (a) and $\delta=0.2 \pi$ (b). The dashed regions show where phase and group velocities are positive, while the continuous line marks the instability threshold (the system is below threshold on the left sides). Negative values of $\delta$ correspond to negative refractive indexes.

velocities can be observed also at the instability threshold of the device (continuous line) depending on the values of $\delta, r \Delta x^{2}$, and $\phi$. The latter is a promising candidate to tune the velocities nonmechanically, for instance by changing the refractive index in the feedback loop.

Whenever the group velocity is non-null, one has to determine whether amplified perturbations of the unstable reference state $E_{0}$ will drift away (convective instability), or will fill the entire system (absolute instability). The convective regime is the one where the control of localized light signals is possible. The nature of the instability is determined by finding the limit of the Green function of the linearized system of equations for large time. The asymptotic local behavior of the perturbation is found by generalizing the saddle point technique developed in $[6,13]-$ the details will be reported elsewhere. In Fig. 1(d) we show an example of thresholds of convective (I) and absolute (II) instabilities; for any choice of parameters there are windows of convective instability before reaching the lasing thresholds. By using the information in Figs. 1 and 2 and Eqs. (3) and (4) we can determine linear amplification, direction of propagation, and spatial chirping of any light spot in the transverse plane.

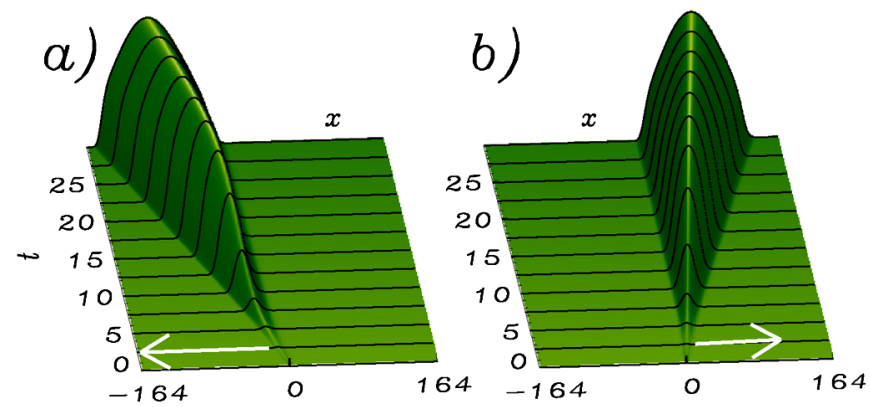

FIG. 3 (color online). Spatiotemporal diagram for the field intensity $|E|^{2}$ starting from a small Gaussian perturbation of the vanishing state $E_{0}$, obtained by numerical simulation of Eqs. (5). Parameters: $\mu=0.98, \theta=0.2, \delta=0.49 \pi, r=0.5$, $\Delta x=1$ (coupling each point with a shifted one on the right) and $\phi=\pi / 2$ (a), $\phi=-\pi / 2$ (b).
In order to check to what extent the linear analysis we reported predicts the dynamics of the full nonlinear device we consider the standard model for class $A$ lasers, obtained from Eqs. (1) with

$$
\left.g_{1}=-(1+i \theta-N) E, \quad N=\mu /\left(1+|E|^{2}\right)\right]
$$

with the usual parameters $\theta$ for the detuning with respect to the medium resonance, and $\mu$ for the pump [15]. The dispersion relation for the field perturbations around the homogeneous steady state $E_{0}=0$ are given by Eq. (2) with $\beta=\mu-1-i \theta$. Numerical simulations confirm the predicted thresholds, in agreement with the stability diagrams in Fig. 1. Moreover, the wave numbers dynamically selected and the velocities are well approximated by those obtained from linear dispersion. In view of the applications, it is interesting to see the dynamics of local perturbation of the homogeneous state: In Fig. 3 we demonstrate the ability of steering and amplifying beams in the convective region; furthermore, one or both the signs of phase and group velocities can be changed with the proper parameters choice [Figs. 3(a) and 3(b)], consistently with predictions presented in Fig. 2. Numerical simulations also confirm the possibility of chirping; the phase of the field shows indeed a spatial dependent modulation.

Special attention needs the case $|\phi|=\pi$, where a small spot of light is amplified and splits in two separate spots traveling in opposite directions as shown in Fig. 4(a). Both positive and negative wave vectors with values around the critical ones are selected and then separate, moving in opposite regions of the beam area. Our analysis for $|\phi|=$ $\pi$ gives $\omega_{R}(k)=\omega_{R}(-k)$ but, in general, $\omega_{I}(k) \neq$ $\pm \omega_{I}(-k)$. This is important because in order to see a propagating stripe, for instance $E \propto \cos (k x+\omega t)$, it would be necessary to have an antisymmetric dispersion $\omega_{I}(k)$ and the simultaneous instability of both positive and negative wave numbers. This would guarantee that the interfer-

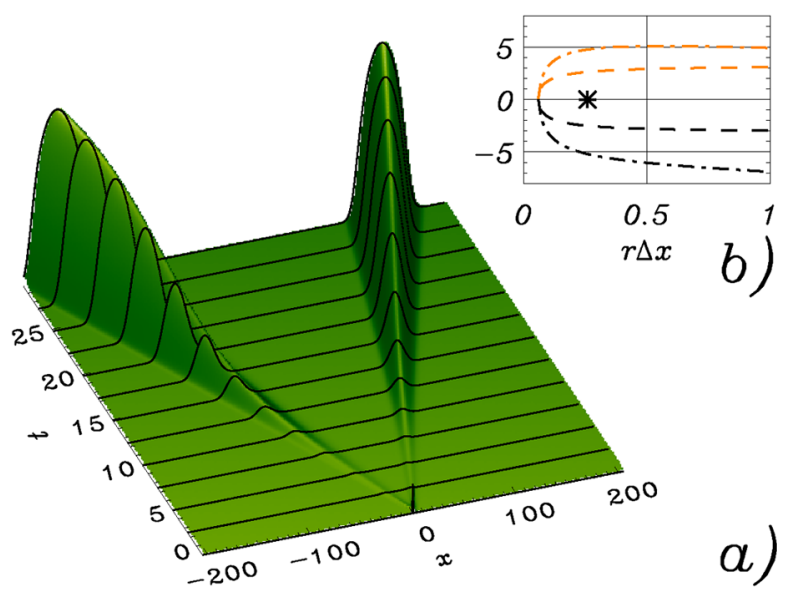

FIG. 4 (color online). (a) Evolution of a Gaussian perturbation as in Fig. 3 but for $\phi=\pi$. (b) Phase (dashed lines) and group (dashed-dotted lines) velocities. The upper (lower) curves are the velocities for $k_{c}\left(-k_{c}\right)$. For $r \Delta x>0.26$ (star point) the homogeneous state is unstable. 
ing waves $k$ and $-k$ have the same velocities. As shown in Fig. 4(b) this is not the case for off-axis feedback: the phase and group velocities of opposite waves with critical wave numbers have opposite signs, and in the diffraction limit $\delta \rightarrow \pi / 2$ both velocities are odd functions of $k_{c}$. Therefore, even if for $\phi=\pi$ both $+k_{c}$ and $-k_{c}$ are unstable, from the linear analysis we do not expect intensity stripe patterns above threshold. The existence of exact traveling phase patterns as well as the lack of intensity waves are also known in lasers without feedback [14]. The novelty here is the prediction of a state in which two waves with wave vectors $\pm k$ travel apart with opposite velocities. In spite of the definite direction associated to the break of reflection symmetry due to two-point nonlocality, both transverse direction of propagation are equally linearly amplified. This is another peculiar effect induced by the combination of diffraction and nonlocality. As shown in Fig. 4(a), numerical simulations of the model (5) for $|\phi|=$ $\pi$ agree fairly well with these predictions. Even if the linear amplification of both waves has the same strength, one wave is nonlinearly favored over the other so that a slightly larger intensity and size of the packet are found on one side with respect to the other, depending on the sign of the shift. As a matter of fact, one mode in the far field is more intense than the other, similar to what is found in systems with drift [16]. We also note that in this case only the Green function correctly characterizes the convective or absolute nature of the instability. The standard evaluation of the instability solely in terms of the velocities of the external fronts of a perturbation would erroneously describe the convective instability as absolute. We have seen, in fact, that here a Gaussian perturbation splits into two wave packets with the external fronts moving in opposite directions, as is usually the case for absolute instabilities, even if the signal eventually decays between the external fronts.

In conclusion, we have reported a general analysis of the effects of off-axis feedback in a large class of optical cavities and gain media, and shown the threshold dependence on two-point nonlocality, diffusion, and positive as well as negative diffraction. The possibility to observe traveling waves at the onset of the instability in media with fast relaxation of the polarization is an important effect of nonlocality, that induces the modulations character of the instability. We have determined the convective and absolute threshold extending our analysis of purely diffusive systems [6]. In the presence of nonlocality, phase and group velocities of optical fields can be easily tuned to parallel or opposite directions, which enable steering and spatial chirping. Surprisingly, for a particular phase of the feedback loop $(\phi=\pi)$ we have found the simultaneous presence of waves traveling apart. The effect is almost symmetrical in the positive and negative directions, even if the off-axis feedback introduces a directional coupling in the transverse plane. The possibility of amplifying an initial spot of light, control its velocity and spatial chirping, and even split it in two counterpropagating signals makes cavities with off-axis feedback a promising candidate in view of applications in all-optical communications based on the control of light signals, such as optical triggering, switching, routing, delay lines, beam recovery and steering, and in manipulation of microparticles. Finally, our theoretical results formally apply to a broad class of devices and similar effects can be observed for localized perturbations of any nonlocal and spatially extended system near the onset of oscillations.

Funding from MEC ("Ramon y Cajal" contract and SICOFIB project), from Govern Balear (No. PROGECIB-5A), and from the European project FunFACS are acknowledged.

*roberta@ifisc.uib.es

[1] F. T. Arecchi, S. Boccaletti, and P. Ramazza, Phys. Rep. 318, 1 (1999).

[2] R. Lang and K. Kobayashi, IEEE J. Quantum Electron. 16, 347 (1980); A. Argyris et al., Nature (London) 437, 343 (2005).

[3] P. L. Ramazza, S. Ducci, and F. T. Arecchi, Phys. Rev. Lett. 81, 4128 (1998); S. Rankin, E. Yao, and F. Papoff, Phys. Rev. A 68, 013821 (2003); L. Pastur, U. Bortolozzo, and P. L. Ramazza, Phys. Rev. E 69, 016210 (2004).

[4] J.P. Seipenbusch, T. Ackemann, B. Schäpers, B. Berge, and W. Lange, Phys. Rev. A 56, R4401 (1997).

[5] E. Louvergneaux, C. Szwaj, G. Agez, P. Glorieux, and M. Taki, Phys. Rev. Lett. 92, 043901 (2004).

[6] F. Papoff and R. Zambrini, Phys. Rev. Lett. 94, 243903 (2005).

[7] M. C. Cross and P. C. Hohenberg, Rev. Mod. Phys. 65, 851 (1993).

[8] G. Giacomelli and A. Politi, Physica D (Amsterdam) 117, 26 (1998).

[9] A. Dunlop, W. J. Firth, and E. M. Wright, Opt. Commun. 138, 211 (1997).

[10] P. Coullet, L. Gill, and F. Rocca, Opt. Commun. 73, 403 (1989).

[11] P. Kockaert, P. Tassin, G. Van der Sande, I. Veretennicoff, and M. Tlidi, Phys. Rev. A 74, 033822 (2006), and references therein.

[12] In the case of nonlinear feedback $\left[f\left(|E|^{2}\right) E\right]_{\Delta x}$, the Eq. (2) needs to be considered with with $r e^{i \phi}=f(0)$.

[13] R. Zambrini and F. Papoff, Phys. Rev. E 73, 016611 (2006).

[14] P. K. Jakobsen et al., Phys. Rev. A 49, 4189 (1994).

[15] J.R. Tredicce, F. T. Arecchi, G.L. Lippi, and G.P. Puccioni, J. Opt. Soc. Am. B 2, 173 (1985); M. Brambilla, L. A. Lugiato, F. Prati, L. Spinelli, and W. J. Firth, Phys. Rev. Lett. 79, 2042 (1997).

[16] R. Zambrini and M. San Miguel, Phys. Rev. A 66, 023807 (2002); R. Zambrini, M. San Miguel, C. Durniak, and M. Taki, Phys. Rev. E 72, 025603(R) (2005). 